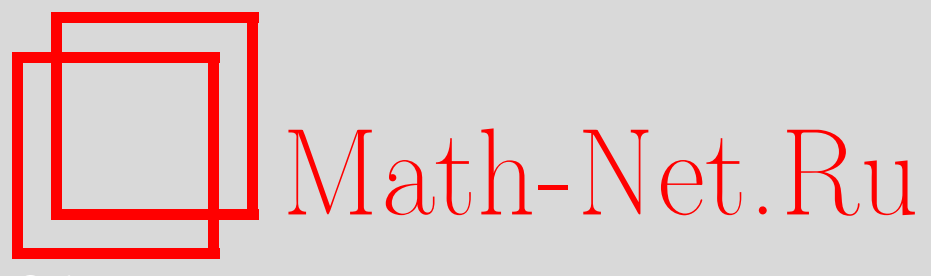

С. А. Теляковский, Оценка одновременного приближения функций и их производных суммами Фурье, $M a$ тем. заметки, 2011, том 90, выпуск 3, 478-480

DOI: https://doi.org/10.4213/mzm9056

Использование Общероссийского математического портала Math-Net.Ru подразумевает, что вы прочитали и согласны с пользовательским соглашением http://www . mathnet.ru/rus/agreement

Параметры загрузки:

IP : 54.237 .59 .107

26 апреля 2023 г., 15:24:00

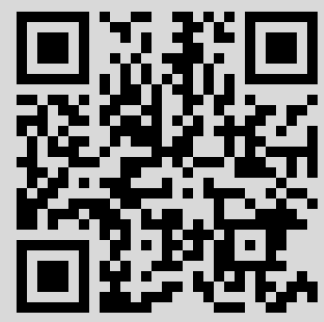




\section{Оценка одновременного приближения функций и их производных суммами Фурье}

\section{С. А. Теляковский}

В [1] рассматривалась следующая задача об одновременном приближении в $C$ суммами Фурье функций класса $W^{r} H[\omega]$ и их производных.

Для набора $m$ целых чисел $0 \leqslant k_{1}<k_{2}<\cdots<k_{m} \leqslant r$ требуется выяснить поведение при $n \rightarrow \infty$ величины

$$
\mathscr{E}_{n}^{(m)}\left(W^{r} H[\omega], S_{n}, x\right):=\sup _{f \in W^{r} H[\omega]} \sum_{i=1}^{m} \frac{1}{n^{k_{i}}}\left|f^{\left(k_{i}\right)}(x)-S_{n}\left(f^{\left(k_{i}\right)}, x\right)\right| .
$$

Заметим, что случай $k_{1}>0$ легко сводится к случаю $k_{1}=0$. Кроме того, в $(1)$ достаточно рассматривать какое-либо одно значение $x$, например, $x=0$. Поэтому в дальнейшем $x$ писать не будем.

В настоящей заметке говорится о величинах $\mathscr{E}_{n}^{(m)}$ только для классов $W^{r}$ ввиду того, что будет использоваться оценка (4), относящаяся к классам $W^{r}$. Для классов $W^{r} H[\omega]$ подобный результат не известен.

В [1] анонсирована оценка

$$
\mathscr{E}_{n}^{(m)}\left(W^{r}, S_{n}\right)=\frac{4}{\pi^{2}} \sqrt{m_{0}^{2}+m_{1}^{2}} \frac{\log n}{n^{r}}+O\left(\frac{1}{n^{r}}\right),
$$

где $m_{0}$ и $m_{1}$ - количество четных и соответственно нечетных чисел среди $k_{1}, k_{2}, \ldots, k_{m}$.

В [2] и [3; § 2.7] публикуются доказательства оценки (2).

Однако, в этих доказательствах проводятся оценки величин $\mathscr{E}_{n}^{(m)}$ только сверху, а об оценках снизу не сказано ни слова. Между тем, в подобных задачах наибольшую трудность обычно представляет получение именно оценок снизу.

Кроме того, там не прослеживался характер зависимости оценок относительно параметров.

Поскольку в этой задаче имеется много параметров, попытка получения в общем случае оценок сверху и снизу, равномерных относительно всех параметров, обречена на неудачу. Но желание иметь оценки, равномерные относительно существенного в этой задаче параметра $m$, оправдано.

Анализ упомянутых доказательств из [2] и [3] показывает, что там фактически установлена равномерная относительно всех параметров оценка

$$
\mathscr{E}_{n}^{(m)}\left(W^{r}, S_{n}\right) \leqslant \frac{4}{\pi^{2}} \sqrt{m_{0}^{2}+m_{1}^{2}} \frac{\log n}{n^{r}}+O\left(\frac{m}{n^{r}}\right) .
$$

Отметим, что оценка (3) нетривиальна, если среди $k_{1}, k_{2}, \ldots, k_{m}$ есть числа разной четности.

Приведем пример, когда удается найти асимптотику величины $\mathscr{E}_{n}^{(m)}\left(W^{r}, S_{n}\right)$.

Пусть $m=2$ и $k_{1}=0, k_{2}=r$. Воспользуемся оценкой из [4]

$$
\sup _{f \in W^{r}}\left\|f(x)-S_{n}(f, x)\right\|_{C}=\frac{4}{\pi^{2}} \frac{1}{n^{r}} \log \frac{n}{\min (n, r+1)}+O\left(\frac{1}{n^{r}}\right),
$$

Работа выполнена при поддержке Российского фонда фундаментальных исследований (грант № 11-01-00417) и программы "Ведущие научные школы" (грант № НШ-65772.2010.1). 
равномерной относительно всех параметров. Так как

$$
\sup _{f \in W^{r}}\left\|f^{(r)}(x)-S_{n}\left(f^{(r)}, x\right)\right\|_{C}=\sup _{|f| \leqslant 1}\left\|f(x)-S_{n}(f, x)\right\|_{C}=\frac{4}{\pi^{2}} \log n+O(1),
$$

в рассматриваемом случае

$$
\mathscr{E}_{n}^{(2)}\left(W^{r}, S_{n}\right)=\frac{4}{\pi^{2}} \frac{1}{n^{r}} \log n+O\left(\frac{1}{n^{r}} \log \frac{n}{\min (n, r+1)}+\frac{1}{n^{r}}\right) .
$$

В частности, при $r>n / 2$

$$
\mathscr{E}_{n}^{(2)}\left(W^{r}, S_{n}\right)=\frac{4}{\pi^{2}} \frac{1}{n^{r}} \log n+O\left(\frac{1}{n^{r}}\right) .
$$

Точно так же с помощью оценки (4) устанавливается следующее утверждение.

Если $k_{1}=0$, а все остальные числа $k_{2}, \ldots, k_{m}$ больше $n / 2$, то равномерно относительно всех параметров

$$
\mathscr{E}_{n}^{(m)}\left(W^{r}, S_{n}\right)=\frac{4}{\pi^{2}} \frac{1}{n^{r}} \log n+O\left(\frac{m}{n^{r}}\right) .
$$

Использованные здесь рассуждения показывают, что в (3) $m_{0}$ и $m_{1}$ можно заменить на количество четных и нечетных чисел среди $k_{1}, k_{2}, \ldots, k_{m}$, которые меньше $n / 2$.

Заметим, что оценки сверху, которые в этих случаях дает формула (3), являются более грубыми.

Приведем еще пример, когда с помощью (4) легко получить оценку, более точную, чем (3).

Пусть $r$ - нечетное число и в качестве $k_{i}$ взяты все четные числа, не превосходящие $r$, т.е. числа

$$
0,2, \ldots, 2\left[\frac{r}{2}\right] \text {. }
$$

Введем обозначение $p:=[r / 2]$. Тогда $r=2 p+1$ и $m=p+1$. Пусть $n \geqslant r$.

С помощью (4) приходим к оценке

$$
\mathscr{E}_{n}^{(m)}\left(W^{r}, S_{n}\right) \leqslant \frac{4}{\pi^{2}} \frac{1}{n^{r}} \sum_{i=1}^{m} \log \frac{n}{\min \left(n, r-k_{i}+1\right)}+O\left(\frac{m}{n^{r}}\right) .
$$

Рассмотрим сумму

$$
\sum_{i=1}^{m} \log \frac{n}{\min \left(n, r-k_{i}+1\right)}=m \log n-\sum_{i=1}^{m} \log (r-2(i-1)+1) .
$$

Имеем

$$
\begin{aligned}
\sum_{i=1}^{m} \log (r-2(i-1)+1) & =\sum_{i=1}^{m} \log (2 p-2 i+4) \\
& =m \log 2+\sum_{i=1}^{p+1}(p-i+2)=m \log 2+\log (p+1) .
\end{aligned}
$$

С помощью формулы Стирлинга получаем

$$
\log (p+1) !=(p+1) \log (p+1)+O(p+1) .
$$

Объединив эти оценки, видим, что

$$
\sum_{i=1}^{m} \log \frac{n}{\min \left(n, r-k_{i}+1\right)}=m \log n-m \log m+O(m)=m \log \frac{n}{m}+O(m),
$$


и таким образом, равномерно относительно всех параметров

$$
\mathscr{E}_{n}^{(m)}\left(W^{r}, S_{n}\right) \leqslant \frac{4}{\pi^{2}} \frac{m}{n^{r}} \log \frac{n}{m}+O\left(\frac{m}{n^{r}}\right) .
$$

В [4] доказано, что оценка вида (4) справедлива также для приближений суммами Фурье функций из классов $\bar{W}^{r}$, сопряженных с функциями класса $W^{r}$. Таким образом, оценки вида (5) и (6) имеют место и для аналогично определяемых величин $\mathscr{E}_{n}^{(m)}\left(\bar{W}^{r}, S_{n}\right)$.

При этом числа $k_{1}, k_{2}, \ldots, k_{m}$ могут быть не обязательно целыми. Переносу результатов из [1] на такой случай посвящена работа [5].

До сих пор имелись в виду приближения в $C$ и соответствующие этому случаю классы $W^{r}$ и $\bar{W}^{r}$. Но можно рассматривать такого рода задачи и для приближений в метрике $L$.

Согласно сказанному в [4] аналог оценки (4) справедлив для приближений в $L$ соответствующих классов функций $W_{L}^{r}$. Поэтому для верхних граней

$$
\sup _{f \in W_{L}^{r}}\left\|\sum_{i=1}^{m} \frac{1}{n^{k_{i}}}\left(f^{\left(k_{i}\right)}(x)-S_{n}\left(f^{\left(k_{i}\right)}, x\right)\right)\right\|_{L}
$$

также имеют место оценки, подобные (5) и (6).

Вопрос об одновременном приближении функций и их производных в метрике $L$ рассматривался в [6], но там речь шла не о величинах (7), а о более грубой характеристике о верхних гранях

$$
\sup _{f \in W_{L}^{r}} \sum_{i=1}^{m} \frac{1}{n^{k_{i}}}\left\|f^{\left(k_{i}\right)}(x)-S_{n}\left(f^{\left(k_{i}\right)}, x\right)\right\|_{L},
$$

для которых были анонсированы асимптотические оценки.

Нетрудно видеть, что оценки вида (5) и (6) справедливы и для величин (8).

\section{СПИСОК ЦИТИРОВАННОЙ ЛИТЕРАТУРЫ}

[1] А. И. Степанец, Докл. АН СССР, 254:3 (1980), 543-544. [2] А. И. Степанец, Укр. матем. журн., 33:3 (1981), 356-367. [3] А. И. Степанец, Равномерные приближения тригонометрическими полиномами. Линейные методы, Киев, Наукова думка, 1981. [4] С. А. Теляковский, Матем. заметки, 4:3 (1968), 291-300. [5] Н. Н. Сорич, А. И. Степанец, Матем. заметки, 36:6 (1984), 873-882. [6] Н. Н. Сорич, Укр. матем. журн., 37:2 (1985), 205-211.

\section{С. А. Теляковский}

Поступило Математический институт им. В. А. Стеклова РАН 28.02.2011

E-mail: Satel@mi.ras.ru 